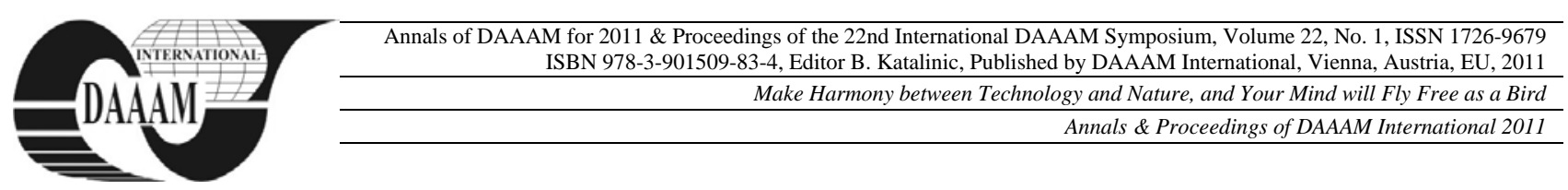

\title{
MATHEMATIC MODEL OF HEAT TRANSFER IN THE IMPULSE SENSOR
}

\author{
JINGA, V[lad]; OROS, R[amona] - G[eorgiana]; SAMOILA, C[ornel] \& URSUTIU, D[oru]
}

\begin{abstract}
This article will present a mathematical model regarding a heat transfer that takes place in a developing new type of gas flowmeter that doesn 't affect at all the section of the pipe through which the gas flows, thus resulting the non usage of a soft correction when it comes to the measured flow.

This fact is caused because of the sensors simpliest way of measuring the gas flow, by using a thermal impulse, generating it and receiving the effects after a gas volume has passed, resulting the heat transfer between the generation element and the gas quantity.
\end{abstract}

Key words: gas flow measurement, heat impulse

\section{INTRODUCTION}

This paper represents an original research to apply an impulse method for a gas flow measurement problem in special conditions.

Most of the flow measurements are using the stationary regime and practically speaking, in many of these cases, there are some interventions in the flow sections with an active element or a special sensor. On that account, for avoiding the above mentioned inconvenient - the alteration of the flow sections - this paper presents a mathematical model proposed to be used for the gas flow measurement in a transitory regime regarding the heat transfer.

The working principle of the developing sensor is simple: a heat pulse is generated in the gas volume that flows through the pipeline and at a specific distance the quantity of the remaining heat in the volume of gas is measured. From those measurements the gas flow can be obtained, because the larger the gas volume that passes the generating pulse point, the smaller the quantity of the remaining heat (temperature) will be at the measuring point.

\section{PHENOMENA DESCRIPTION}

The repeating cycle implemented with the sensor will consist of a pulse generation, increasing the temperature of the whole gas volume regarding a specific lenght in the moment of the generation thus resulting the heat transfer. After that, the heated slice of gas volume will travel along the pipe till the measurement point, simultaneously with this loosing heat because of the dissipation. At the measuring point, the heat loss will be determined, thus resulting the real flow of the gas.

This cycle will be repeated directly depending on the speed of the gas, this being equivalent with the flow. The developing sensor will be used for low flows of gas in the medical domain and also in industrial environment. So because of that, the frequency with which the described cycle will be repeated will be determined experimentally in the moment when the calibration of the sensor will be done.

As for the gas, reporting to a slice that gets the thermal pulse in the heat exchange, the specific volume of the gas will be heated (increasing its temperature) and then slowly it will cool down (decreasing its temperature), like shown in the next figure:

Section $\mathrm{a}-\mathrm{b}$ shown in the figure represents the moment when the volume of gas is flowing in the pipeline before the generation pulse point and the gas has a constant temperature $\mathrm{T}_{1}$.

Section $b-c$ shown in the figure represents the moment after the volume of gas has passed the generation pulse point in the pipeline, got the thermal impulse and the heat exchange occurred thus increasing the temperature of the gas until the maximum, $\mathrm{T}_{2}$.

Section $\mathrm{c}-\mathrm{d}$ shown in the figure represents the moment after the gas volume has reached the maximum temperature due to the heat transfer from the thermal pulse and starts cooling off.

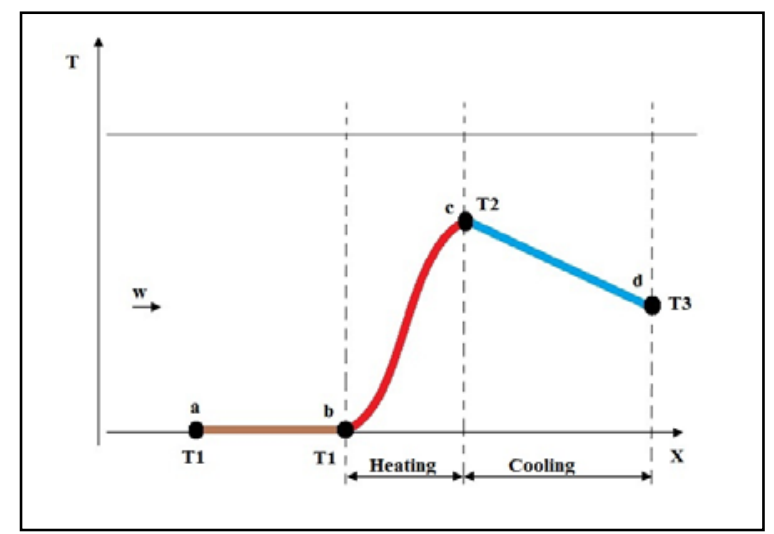

Fig. 1. The heat transfer and the gas temperature evolution

\section{THE HEAT TRANSFER}

The heat transfer in this situation, regarding the phenomena that occurs inside the flow sensor because of the thermal impulse, can be calculated using the method of thermal balance in transient regime with the elementary cube like in the next figure:

$\mathrm{dQ}_{1}$ is the elementary cube heat gained from the walls as a result of generating the thermal impulse. This heat is transferred from the generating pulse element to the gas volume through conduction and is according to the Fourier ecuation:

$$
d Q_{1}=-\lambda \frac{\delta T}{\delta X} \times d S
$$

where dS represents the surface through which the heat exchange takes place.

The used hyphotesis in this case is that in the elementary layer the conduction and the convection are equal according to the convection contour law. 
$\mathrm{dQ}_{2}$ represents the heat quantity accumulated in the considerated gas volume (the elementary cube). Because the gas is moving, the formula for this heat is:

$$
d Q_{2}=C_{p} \times \rho \times d V \times \frac{D T}{D \zeta}
$$

where $C_{p}$ is the gas specific medium heat at constant pressure, $\rho$

is the gas density, $\mathrm{dV}$ is the volume element and DT/D represents the temperature substantial derivative depending on time.

Considering the unidimensional variation hyphothesis, the operator DT/D $\delta$ from relation 2 will be:

$$
\frac{D T}{D \zeta}=\frac{\delta T}{\delta X} \times \frac{\delta X}{\delta \zeta}=\frac{\delta T}{\delta X} \times \omega
$$

so relation 2 will become:

$$
d Q_{2}=C_{p} \times \rho \times d V \times \omega \times \frac{\delta T}{\delta X}
$$

$\mathrm{dQ}_{3}$ represents the heat quantity transmitted to the cooler layers of gas and according to the Fourier ecuation is:

$$
d Q_{3}=-\lambda \frac{\delta}{\delta X} \times\left(T+\frac{\delta T}{\delta X}\right) d S
$$

The ecuation of the thermal balance is, as shown in fig. 2:

$$
\mathrm{dQ}_{1}=\mathrm{dQ}_{2}+\mathrm{dQ}_{3}
$$

Before doing all the replacements in and solving the last relation (6 - the thermal balance equation), heat $1\left(\mathrm{dQ}_{1}\right)$ and 3 $\left(\mathrm{dQ}_{3}\right)$ - the heats transferred through the surface $\mathrm{dS}$ - must be the subject of some Gauss - Ostrogradski transformations, because heat $2\left(\mathrm{dQ}_{2}\right)$ is expressed in volume units. After the Gauss - Ostrogradski transformations, the results are:

$$
\begin{gathered}
d Q_{1}=-\lambda \times \frac{\delta^{2} T}{\delta X^{2}} d V \\
d Q_{3}=-\lambda\left(\frac{\delta^{2} T}{\delta X^{2}}+\frac{\delta^{3} T}{\delta X^{3}}\right) d V
\end{gathered}
$$

After replacing everything in relation 6 and doing all the possible simplifications, the thermal balance equation becomes:

$$
\frac{\delta^{3} T}{\delta X^{3}}=\frac{C_{p} \times \rho \times \omega}{\lambda} \times \frac{\delta T}{\delta X}
$$

Working with the substitution $y=\delta \mathrm{T} / \delta \mathrm{X}$ in realtion 9 , the final form of the thermal balance equation will be:

$$
\frac{\delta}{\delta X}\left(y \frac{\delta y}{\delta T}\right)=\frac{\delta^{3} T}{\delta X^{3}}
$$

The relation between the travelling speed of the gas in the pipeline and its temperature in the moment of heating will be:

$$
\frac{\delta}{\delta X}\left(y \frac{\delta y}{\delta T}\right)=\frac{c_{p} \times \rho \times \omega}{\lambda} \times y
$$

Between two thermal impulses, the gas from the elementary cube will have a cooling off time; comparing to the previous described thermal balance - in the heating situation, in this situation the heating source is no more, so heat $1\left(\mathrm{dQ}_{1}\right)$ is null.

Again, after replacing everything in the relations and doing all the possible simplifications, the thermal balance equation for the cooling period becomes:

$$
\frac{\delta^{2} T}{\delta X^{2}}+\frac{\delta^{3} T}{\delta X^{3}}=\frac{C_{p} \times \rho \times \omega}{\lambda} \times \frac{\delta T}{\delta X}
$$

Repeating the same procedure as for the previous described thermal balance - in the heating situation and doing the substitution $y=\delta \mathrm{T} / \delta \mathrm{x}$ in relation 12 , the dependence between the travelling speed of the gas in the pipeline and the gas temperature in the moment of cooling down will be:

$$
y \frac{\delta y}{\delta T}+\frac{\delta}{\delta X}\left(y \frac{\delta y}{\delta T}\right)=\frac{C_{p} \times \rho \times \omega}{\lambda} \times y
$$

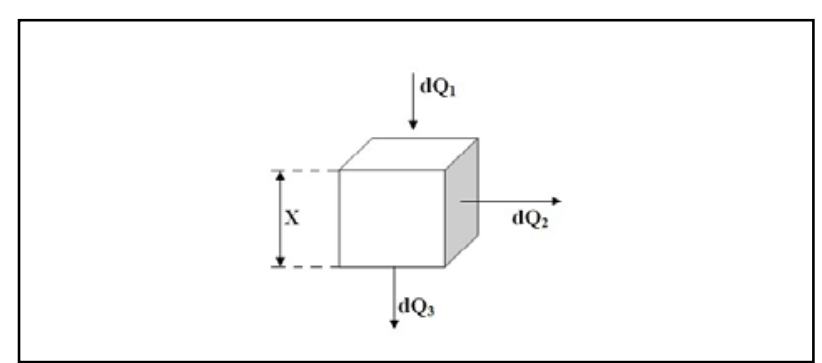

Fig. 2. The elementary cube with all its elements

\section{FUTURE WORK}

The practical development regarding the new flow sensor and the calibration of this flowmeter are desired to be done as soon as possible.

\section{CONCLUSIONS}

1. The above model was designed in the idea of not alterating the measurement section of the flowmeter;

2. The second advantage of this model is the fact that measurements will be possible both in trazient regimes and in stationary ones;

3. The third advantage is the fact that this model will allow measurements for very small values of the flow.

\section{ACKNOWLEDGEMENTS}

This paper is supported by the Sectoral Operational Program "Human Resources Development" (SOP HRD), financed from the European Social Fund and by the Romanian Government under the contract number POSDRU/88/1.5/S/59321 and POSDRU/6/1.5/S/6.

\section{REFERENCES}

Ahmed Al - Salaymeh, Moh`d Sami Ashhab (2005): Modelling of a novel hot wire thermal flow sensor with neural nets under different operating conditions Elsevier

Izguierdo - Gil M. A. (2008): Temperature influence on transport parameters characteristic of Knudsen and Poiseuille flows Elsevier

Koizumi H., Serizawa M. (2008): A micro flowmeter based on the velocity measurement of a locally accelerated thermal flow in an upwardly directed Hagen - Poisseuille flow Elsevier

Rumyantsev A. V., Gus`kov K. V. (2007): Variable - power thermal gas microflowmeters Measurement techniques, Vol. 50, No. 8

Rumyantsev A. V., Gus`kov K. V. (2008): A gas microflowmeter with a specific sensitivity value Instruments and experimental techniques, Vol. 51, No. 1, ISSN 00204412 